\title{
Tension optimization of the 6-DOF cable-driven boat motion simulator
}

\author{
Joonyoung Jang* \\ Mechanical and Aerospace Engineering Department, \\ University of California, San Diego, 9500 Gilman Dr, La \\ Jolla, CA 92093, USA
}

\begin{abstract}
The 6 degrees of freedom cable-driven boat motion simulator has eight cables and a moving platform in replicating the boat motion. The platform is connected to cables and is moved by adjusting the length of the cables. The simulator has more cables than its degrees of freedom is defined as an underdetermined system, which means the tension solution is not unique and can be optimized while controlling the platform to the desired position. Leveraging the equilibrium condition and the dynamic equation of tensegrity systems, linearized equations of motion of the moving platform were derived. A Linear Matrix Inequality (LMI)-based controller design and a convex optimization validated to stabilizing the system and optimizing cable tensions. A simulation model is suggested to verify optimized cable tension solutions and the feasibility of the LMI based controller.
\end{abstract}

\section{CCS CONCEPTS}

- Computing methodologies $\rightarrow$ Modeling and simulation; Simulation evaluation; - Theory of computation $\rightarrow$ Design and analysis of algorithms; Algorithm design techniques; Dynamic programmingInsert your third CCS term here.

\section{KEYWORDS}

cable-driven systems, tension optimization, Linear matrix inequality

\section{ACM Reference Format:}

Joonyoung Jang and Thomas R. Bewley. 2021. Tension optimization of the 6-DOF cable-driven boat motion simulator. In 2021 the 3rd International Conference on Robotics Systems and Automation Engineering (RSAE) (RSAE 2021), May 28-30, 2021, Paris, France. ACM, New York, NY, USA, 8 pages. https://doi.org/10.1145/3475851.3475854

\section{INTRODUCTION}

Vertical take-off and landing (VTOL) drones are ubiquitously used as low-price assets, easy to maneuver. In naval operations, drones can expand the reconnaissance and surveillance range of ships.

\footnotetext{
*This author is with Republic of Korea Navy, 625 Sindoan-myeon, Gyeryong-si, Chungcheongnam-do, Korea, j7jang@eng.ucsd.edu
}

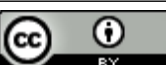

This work is licensed under a Creative Commons Attribution International 4.0 License.

RSAE 2021, May 28-30, 2021, Paris, France

(C) 2021 Copyright held by the owner/author(s)

ACM ISBN 978-1-4503-8846-7/21/05.

https://doi.org/10.1145/3475851.3475854

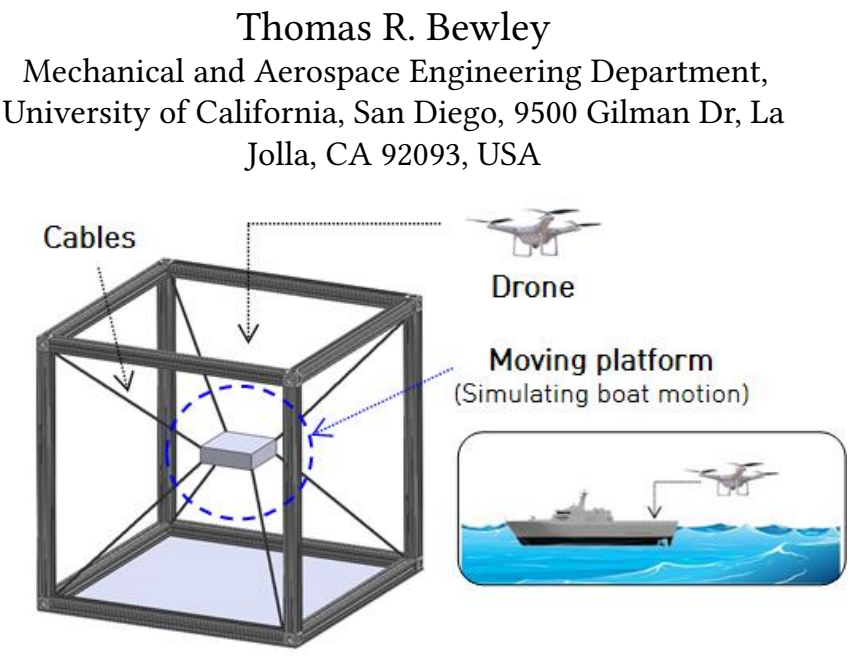

Figure 1: The design of the 6-DOF cable-driven boat motion simulator. Eight cables connect to the moving platform, controlling the length of cables, replicating a boat's motion for at-sea testing drone landing controllers.

However, landing at sea is challenging because the landing platforms are moving by waves. Thus, a boat motion simulator is useful for developing sea landing controllers and confirming drones' landing ability. The boat motion simulator allows for land-based testing, seducing cost and risk [1]. The Stewart platform mechanism provides a 6-DOF capability using six identical stretchable rods and spherical joints. However, the moving platform has a limited workspace range due to its complicated mechanical system [2]. The 3-PSR Parallel mechanism was developed for replicating boat motion [1], but its upper structure obstructs any drone landing capabilities. Motivated by Max Planck's CableRobot Simulator [3], a cable-driven tensegrity system is proposed, as shown in Figure 1. By adjusting the length of cables connected to the moving platform, the platform can be moved in 6-DOF with high acceleration and accuracy [4]. Prior research discussed various challenges with cable-driven systems, including workspace analysis [5, 6], the cable collisions detection algorithm [7]. These cable-driven systems show the benefits of the simplicity of kinematics and its application. A proportional-derivative controller (PD) is used for dynamic control of cable-driven systems [8-10]. However, the PD controller's derivative term amplifies noise in the system and causes actuator oscillations [11].

One significant feature of cable-driven systems is that cables only can pull an object but cannot push it. Thus, cable tensions have to be positive for the robust and stable motion simulations. Specifically, low cable tensions induce slack in cables and potential system failure. High cable tensions can damage the mechanical structure. A cable-driven system having more cables than its DOF 
is called an underdetermined system, in which cable tension solutions are not unique. Although, cable lengths at every state of the platform can be calculated by inverse kinematics [12], optimal tension solutions need to be considered. Other research used the null space of the static equation solution to calculate feasible cable tension solutions $[4,13]$. However, for a dynamically moving platform, tension optimization needs to be performed within the control system. A cable-driven motion simulator can be defined as a tensegrity system with multiple bars and cables. The analysis of the tensegrity system's statics and dynamics is a useful method to characterize the forces in bars and cables [14]. The stabilization of a multi-tethered balloon used the tensegrity analysis to find the optimal design to keep all cables taut even there exist disturbances such as winds [15]. To achieve the tension optimization in the control system, a linearized tensegrity dynamics equation that is in terms of cable tensions was used.

To control the platform motion, the LMI-based controller was designed because it can express a variety of design specifications by setting up constraints [16]. Moreover, the LMI-based control design has less vibrating and guarantees better system response stability than the conventional Linear Quadratic Regulator (LQR) controller design [17].

In this paper, we used the tensegrity system statics and dynamics to analyze a cable-driven boat motion simulator and discussed the optimization of cable tensions. Furthermore, instead of using a PD controller, we applied an LMI-based controller with a linearized system derived from the tensegrity system dynamics. The simulation model was developed to validate the tension solutions and LMI-based controller. This paper is organized as follows. Section II analyzes a cable-driven simulator as a tensegrity system and discusses tension optimization. In Section III, the linearized system is derived from dynamic equations of motions, and the feedback control system is developed. In Section IV, the simulation result is discussed. Section $\mathrm{V}$ is the conclusion.

Tensegrity system analysis

\subsection{Tables equilibrium of the tensegrity system}

Cable-driven systems include cables and bars defined as members. The connecting points of members are defined as nodes. Members and nodes are denoted as the vectors of,

$$
M=\left[\vec{m}_{1}, \cdots, \vec{m}_{p}\right], \quad N=\left[\vec{n}_{1}, \cdots, \vec{n}_{q}\right]
$$

where $p$ and $q$ are the number of the members and nodes, respectively. Each member vector is defined as difference of two connected node vectors, such that $\vec{m}_{i}=\vec{n}_{j}-\vec{m}_{k}$, where $i \in\{1, \cdots, p\}$ and $j, k \in\{1, \cdots, q\}$. Thus, the relation between the member and node vectors can be written by defining the $p \times q$ connectivity matrix $C$ [12],

$$
M=N C^{T}
$$

Each $i$-th row of $C$ corresponds to each $\vec{m}_{i}$ has one entry equal to 1 and one entry equal to -1 , and all other entries equal to zero. Define the sum of gravitational and inertial forces on the nodes as $F$ and the internal forces applied by members as $I$. Let $\vec{d}_{i}$ be a unit member vector, and $\vec{x}_{i}$ be a tensile or compressive force in a member. Then, the unit member and forces can be written [13],

$$
D=\left[\vec{d}_{1}, \cdots, \vec{d}_{p}\right], \quad X=\operatorname{diag}\left(x_{1}, \cdots, x_{p}\right)
$$

The internal forces at each node can be defined in terms of $X$ by using the connectivity matrix, such that,

$$
I=D X C
$$

Using the Newton's law, the forces at each node can be written as,

$$
F=\left[\vec{F}_{1}, \cdots, \vec{F}_{p}\right]=\left(\sum_{i=1}^{Z} m_{i}\left(\vec{a}_{i}+\vec{g}\right) / Z\right)_{p}
$$

where $m_{i}$ is the mass, $\vec{a}_{i}$ is the acceleration vector of $\mathrm{i}$-th member, $\vec{g}$ is the gravitational acceleration vector, $Z$ is the number of nodes $i$-th member has. Figure 2 shows all the forces exerted on a node.

At equilibrium, forces in each node are balanced. The equation can be written,

$$
F=I \quad \rightarrow \quad F=D X C
$$

Recall that $X$ is the diagonal matrix of tension or compression force of each member, indicating that $X$ represents the forces in the bars and cables. Thus, cable tensions can be calculated by solving equation 1) for $X$ [13]. The right-hand side of equation 1) is a linear equation of $\left[x_{1}, \cdots, x_{p}\right]$, so that the equation can be rewritten in the form of a linear matrix equation in terms of vector $\vec{x}=$ $\left[x_{1}, \cdots, x_{p}\right]^{T}$,

$$
A \vec{x}=\vec{f}
$$

where $\vec{f}$ is forces on each node, defined as $\vec{f}=\left[f_{1}, \cdots, f_{p}\right]^{T}$.

\subsection{Tension solution}

Tension solutions can be obtained by solving equation 2). The Singular Value Decomposition (SVD) of the matrix $A=U \Sigma V^{H}$, where $U$ and $V$ are unitary matrices, $\Sigma$ is the diagonal matrix consisting of singular values of $A$, and $V^{H}$ defines the conjugate transpose of matrix $V$. Assuming that the rank of $A$ is $r$, then by definition, the SVD of $A$ becomes [18],

$$
\left.A=\left[\underline{U}_{m \times r} \quad \bar{U}_{m \times(m-r)}\right]\left[\begin{array}{cc}
\underline{\Sigma}_{r \times r} & 0 \\
0 & 0
\end{array}\right] \underline{[}_{n \times r} \quad \bar{V}_{n \times(n-r)}\right]^{H}
$$

The underdetermined system, which has more cables than its DOF, has an infinite number of solutions for $\vec{x}$. Recall that $\bar{V}$ is the null space of $A$ and (2) can be rewritten,

$$
A(\vec{x}+\bar{V} h)=\vec{f}
$$

where $h$ is an arbitrary vector. The solution $\vec{x}$ can be obtained by,

$$
\vec{x}=A^{+} \vec{f}+\bar{V} h
$$

where $A^{+}$is the pseudo-inverse matrix of $A . \vec{x}$ is the internal force vector in each bar and cable defined as,

$$
\vec{x}=\left[\begin{array}{l}
\beta \\
\tau
\end{array}\right]
$$

where $\beta$ is the force in the bars, and $\tau$ is cable tensions. 


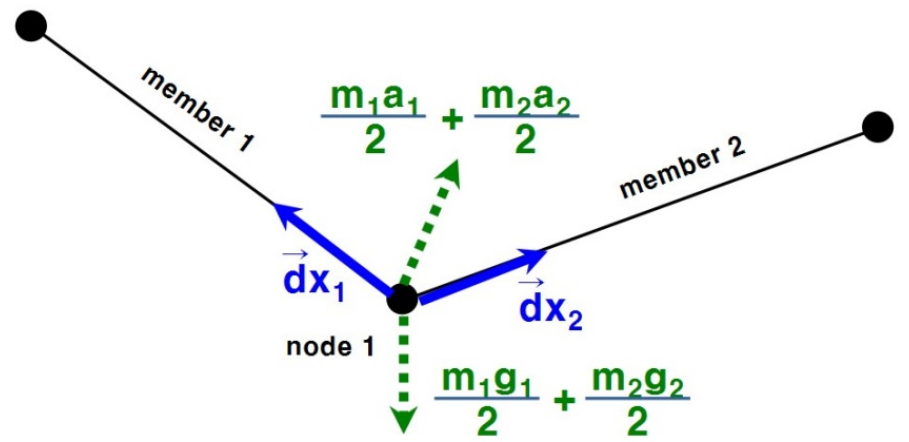

Figure 2: Free body diagram of the tensegrity system. Two members are connected to node 1 and each member has two nodes. At node 1, the total force is the sum of the gravitation and inertial forces of two members. The forces of members are exerted uniformly at each node. The internal force (blue) is the sum of tension or compression forces applied by members $\left(\overrightarrow{d_{1}} x_{1}, \overrightarrow{d_{2}} x_{2}\right)$.

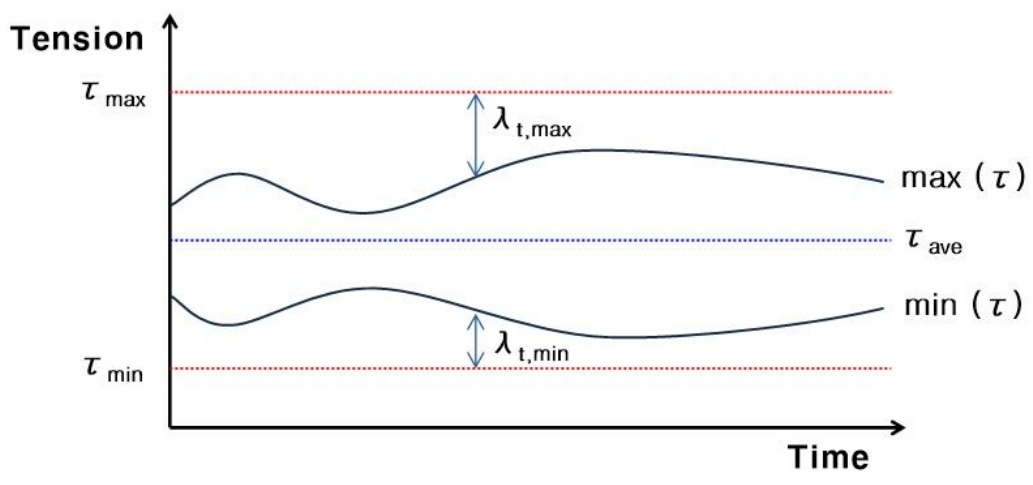

Figure 3: A typical tension graph as in time. The red dashed lines, $\left(\tau_{\min }, \tau_{\max }\right)$, are tension limits the blue dashed line, $\left(\tau_{a v e}\right)$, is the mean value of tension limits. The black solid lines, $(\min (\tau), \max (\tau))$, are the minimum and the maximum tensions and $\left(\lambda_{t, \min }, \lambda_{t, \max }\right)$ are the tension margins at time $t$.

\subsection{Tension optimization}

As shown in Figure 3, cable tensions changes in time as the platform moves to the desired position. Cable tensions need to be maintained in the boundary to prevent cables being slack or damaged, such that,

$$
\tau_{\min } \leq \tau \leq \tau_{\max }
$$

where $\tau_{\min }$ is the minimum tension limit, and $\tau_{\max }$ is the maximum tension limit.

Defining the lower and the upper bound tension margins, $\left(\lambda_{t, \text { min }}, \lambda_{t, \text { max }}\right)$ as the difference between the tension limits $\left(\tau_{\min }, \tau_{\max }\right)$, and the minimum and the maximum tensions $(\min (\tau), \max (\tau))$. A system with larger tension margins has more capability of moving the platform to the tension limits. Tensions are required to be optimized around the mean value of the minimum and the maximum tension limits to maximize the tension margins. Recall that $\bar{V}$ is the null space of $A$, so that variable $h$ can be optimized. Maximizing tension margins is equivalent to minimizing the standard deviation of tensions relative to $\tau_{a v e}$. Leveraging the equation of standard deviation, the optimization problem can be written as a convex problem as follows,

$$
\text { minimize } \sqrt{\frac{\sum_{i=1}^{m}\left(\tau_{i}-\tau_{a v e}\right)^{2}}{b}}
$$

subject to $\tau_{\min } \leq \tau \leq \tau_{\max }$

$$
\tau_{\text {ave }}=\left(\tau_{\min }+\tau_{\max }\right) / 2
$$

$\vec{x}=A^{+} \vec{f}+\bar{V} h$

where $b$ is the number of cables. The optimal value $h^{*}$ is determined by solving equation 5) [19]. To solve such a problem, a convex problem solver, such as CVX is needed [20]. The optimized force vector $\vec{x}^{*}$ can be calculated by using equation 3 ). Recall that $\vec{x}^{*}=\left[\beta_{1}{ }^{*}, \cdots, \beta_{a}{ }^{*}, \tau_{1}{ }^{*}, \cdots, \tau_{b}{ }^{*}\right]^{T}$ with $a$, the number of bars, and $b$, the number of cables. Finally, the optimized tension solution becomes,

$$
\tau^{*}=\left[\tau_{1}^{*}, \cdots, \tau_{b}{ }^{*}\right]^{T}
$$

\subsection{Cable length equation}

Note that the platform can be moved by adjusting the length of cables. Namely, the experimental system needs motors to change 
the length of cables, and motor signals are derived by cable length changes. Thus, cable lengths need to be calculated to actuate the motors. Cable lengths, U, can be determined by using the following equation [13],

$$
U=\frac{\kappa|\vec{l}|}{\tau+\kappa}
$$

where $\kappa$ is the cable stiffness, $|1|$ is the distance between cable attachment points on the platform and the starting points of cables. The optimized cable tension was derived by (6), then the corresponding cable length $U^{*}$ can be determined by applying $\tau^{*}$ to equation 7 ).

\section{DYNAMICS AND CONTROLS}

\subsection{Dynamic equation}

The translational equations of motion of the platform can be calculated by solving Newton's equation,

$$
m \ddot{r}=\vec{f}_{c}+\vec{g} \rightarrow \ddot{r}=\left(\vec{f}_{c}+\vec{g}\right) / m
$$

where $\ddot{r}$ is the acceleration vector of the platform, $\vec{f}_{c}$ is the sum of the forces applied by cables connected to the platform, and $\vec{g}$ is the gravitational force. Similarly, the rotational equations of motion of the platform are solved using Euler's rotation equation,

$$
I_{c m} \dot{\omega}+\omega \times I_{c m} \omega=\vec{t}_{c} \quad \rightarrow \quad \dot{\omega}=\left(\vec{t}_{c}-\omega \times I_{c m} \omega\right) / I_{c m}
$$

where $\dot{\omega}$ is the time derivative of angular velocity vector, $\vec{t}_{c}$ is the total torque from cable tensions, and $I_{c m}$ is the moment of inertia of the platform. By definition, the torque, $\vec{t}_{c}$ can be written as,

$$
\vec{t}_{c}=\vec{n}^{B} \times\left(\vec{f}_{c}\right)^{B}
$$

where $\vec{n}^{B}$ and $\left(\vec{f}_{c}\right)^{B}$ are the node and force vectors in the body frame. The body frame is anchored in the center of the platform. The force vector in the body frame can be written in terms of the force vector in the original frame,

$$
\left(\vec{f}_{c}\right)^{B}=R^{-1} \vec{f}_{c}
$$

where $R$ is the 3-2-1 Tait-Bryan rotation matrix and $\vec{f}_{c}$ is the force vector in the original frame. The torque of the platform becomes,

$$
\vec{t}_{c}=\vec{n}^{B} \times R^{-1} \vec{f}_{c}
$$

\subsection{Linearization}

The translational equation of motion, equation 8), and rotational equation of motion equation 9), can be converted to matrix form as [21],

$$
\left[\begin{array}{l}
\vec{f}_{c} \\
\vec{t}_{c}
\end{array}\right]+\left[\begin{array}{c}
\vec{g} \\
0
\end{array}\right]=\left[\begin{array}{cc}
m I & 0 \\
0 & I_{c m}
\end{array}\right]\left[\begin{array}{c}
\ddot{r} \\
\dot{\omega}
\end{array}\right]+\left[\begin{array}{c}
0 \\
\omega \times I_{c m} \omega
\end{array}\right]
$$

Rearranging the equation about $(\ddot{r}, \dot{\omega})$ yields,

$$
\left[\begin{array}{c}
\ddot{r} \\
\dot{\omega}
\end{array}\right]=\left[\begin{array}{cc}
m I & 0 \\
0 & I_{c m}
\end{array}\right]^{-1}\left(\left[\begin{array}{l}
\vec{f}_{c} \\
\vec{t}_{c}
\end{array}\right]+\left[\begin{array}{l}
\vec{g} \\
0
\end{array}\right]-\left[\begin{array}{c}
0 \\
\omega \times I_{c m} \omega
\end{array}\right]\right)
$$

Defining the state vector $X=[\boldsymbol{r} \dot{\boldsymbol{r}} \Theta \omega]$ with $\Theta$, the rotation angles, equation 10) can be written as,

$$
\dot{X}=\left[\begin{array}{c}
\dot{\boldsymbol{r}} \\
\ddot{r} \\
\omega \\
\dot{\omega}
\end{array}\right]=\left[\begin{array}{c}
\dot{\boldsymbol{r}} \\
(m I)^{-1}\left(\vec{f}_{c}+\vec{g}\right) \\
\omega \\
I_{c m}{ }^{-1}\left(\vec{t}_{c}-\omega \times I_{c m} \omega\right)
\end{array}\right]
$$

Recall that $\vec{f}_{c}$ is the sum of the forces on the platform applied by cables. Thus, $\vec{f}_{c}$ can be written as,

$$
\vec{f}_{c}=\sum_{k=1}^{b} \vec{f}_{c, k}
$$

where $\vec{f}_{c, k}$ is the force applied by k-th cable. Let all the forces at nodes applied by cables be $F_{c}=\left[\vec{f}_{c, 1}, \cdots, \vec{f}_{c, b}\right] . F_{c}$ can be described in terms of cable tensions, $\tau$ [13],

$$
F_{c}=D_{c} T C_{c}
$$

where $D_{c}$ is the unit cable vector set, $T=\operatorname{diag}\left(\tau_{1}, \cdots, \tau_{b}\right)$ is the diagonal matrix of tensions, and $C_{c}$ is the cable connectivity matrix. Note that $D_{c}$ is determined by the platform state $X$, and $\tau$ is the function of cable length $U$ as defined in equation 7). Thus, $\dot{X}$ is a function of $X$ and $U$, denoted as $\dot{X}=f(X, U)$. Similarly, define the output as $Y=\hat{C} X$. The output of the system is the state of the platform. Hence, $\hat{C}$ is an identity matrix and the output vector can be written as,

$$
Y=X=g(X, U)
$$

Finally, the system realization can be determined by Jacobian matrices as follows [22],

$$
\begin{array}{ll}
\hat{A}=\frac{\partial f\left(X_{e q}, U_{e q}\right)}{\partial X}, & \hat{B}=\frac{\partial f\left(X_{e q}, U_{e q}\right)}{\partial U} \\
\hat{C}=\frac{\partial g\left(X_{e q}, U_{e q}\right)}{\partial X}, & \hat{D}=\frac{\partial g\left(X_{e q}, U_{e q}\right)}{\partial U}
\end{array}
$$

$X_{e q}$ and $U_{e q}$ are the position and cable length at the equilibrium, relatively. Specifically, $X_{e q}$ is the desired position, and the $U_{e q}$ is cable lengths calculated by equation 7) corresponding to optimized tension $\tau^{*}$.

\subsection{Feedback control model}

Leveraging the linearized system, the feedback control system can be modeled as shown in Figure 4. The system input is the desired position and the output is the platform position. The algorithm of the system consists of three steps as follows.

2.3.1 LMI-based controller. Based on the system realizations calculated by equation 11 ), the controller gain $K$ can be designed. In Lyapunov theory, the linearized feedback system is stable if and only if there exists a positive definite symmetric matrix, $P>0$, such that [23],

$$
(\hat{A}+\hat{B} K)^{T} P+P(\hat{A}+\hat{B} K)<0
$$

where $\hat{A}$ and $\hat{B}$ are system realizations derived by equation 11$)$. This inequality, called Lyapunov inequality, is a form of an LMI [23]. Introducing variables $Q=P^{-1}$, and $L=K P^{-1}$, equation 12) can be rewritten as [24],

$$
Q \hat{A}^{T}+\hat{A} Q+L^{T} \hat{B}^{T}+\hat{B} L<0
$$

Obtaining $Q$ and $L$ by solving the inequality, one can find a feasible controller gain that stabilizes the system, such that $K=L P$ [25]. Equation 13) can be solved by an LMI solver such as YALMIP [26]. The LMI-based controller takes the position error $\tilde{X}=X-X_{e q}$ and generates the system input $\tilde{U}=K \tilde{X}$. Since the equilibrium platform position $X_{e q}$ and cable length $U_{e q}$ change in time, the 


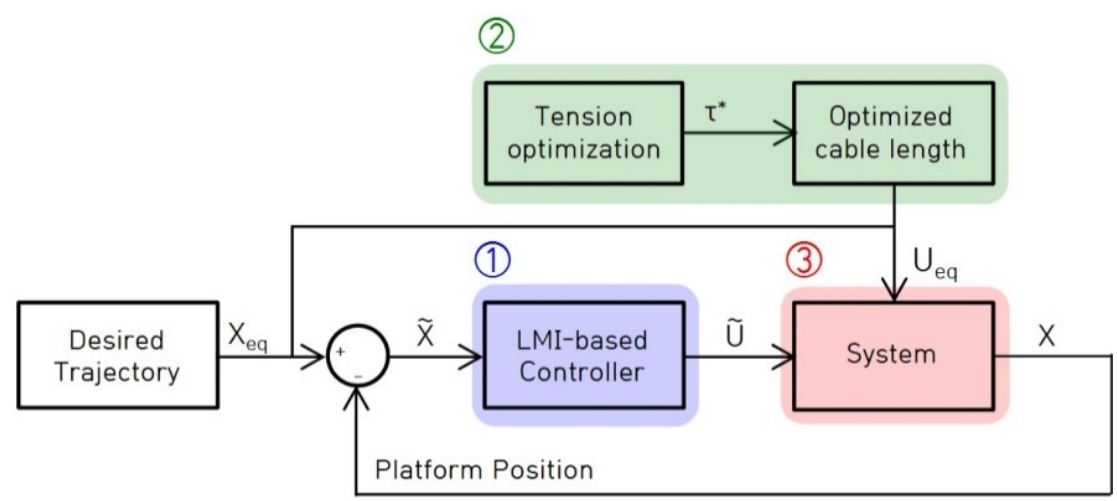

Figure 4: Feedback control system diagram.

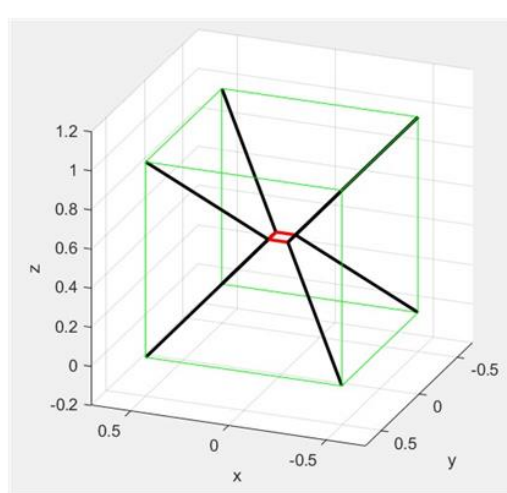

(a) $t=0 s$

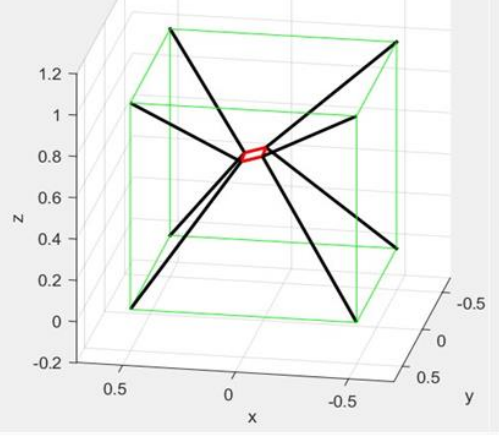

(b) $t=3 s$

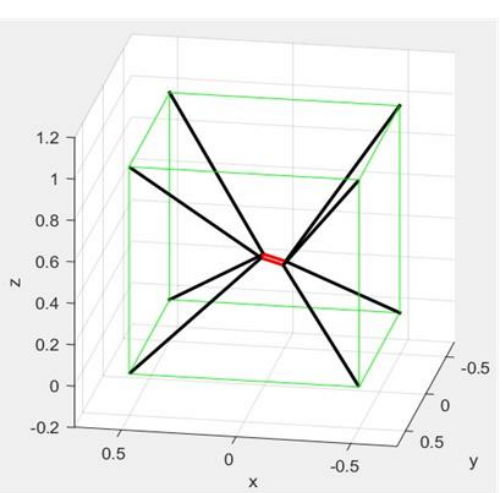

(c) $\mathrm{t}=7 \mathrm{~s}$

Figure 5: The figure of the 6-DOF cable-driven simulation model. The outer structure (green) is a cube. Cables (black) are connected to a rectangular shape moving platform (red). The platform moves in 6-DOF motion over time

system is defined as a time-variant system. Thus, the controller gain is required to be updated at every time step.

2.3.2 Optimization of cable tensions. The optimal variable $h^{*}$ and the optimized cable tension $\tau^{*}$ was derived in Section 2.3. The corresponding cable length $U^{*}$ represents the optimal cable length at the equilibrium point, such that $U_{e q}=U^{*}$. The equilibrium cable length $U_{e q}$ is used to obtain the linearized system denoted at equation 11).

2.3.3 System. The system realization is derived from equation 11) and the controller was designed from equation 13). Thus, the feedback state-space can be written,

$$
\begin{gathered}
\frac{d \tilde{X}}{d t}=\hat{A} \tilde{X}+\hat{B} \tilde{U} \\
\frac{d \tilde{X}}{d t}=(\hat{A}+\hat{B} K) \tilde{X}
\end{gathered}
$$

The output of the state-space model is $\tilde{X}$. Since $\tilde{X}$ is the difference between the desired position and the current position, the platform position becomes $X=\tilde{X}+X_{e q}$ and $X$ is sent back to the system as a feedback.

\section{SIMULATION RESULT}

\subsection{Simulation model description}

The purpose of the simulation model is to verify the tension solutions and the feasibility of the LMI-based controller. As shown in Figure 5, the edges of the outer structure cube are $1 \mathrm{~m}$. The simulation model has eight cables connected to the moving platform. The mass of the platform is $1 \mathrm{~kg}$, and its mass center is the center of the platform. The platform is $0.1 \mathrm{~m}$ flat square plate and has four attachment points in the corners. The minimum tension limit was set to $10 \mathrm{~N}$ and the maximum as $100 \mathrm{~N}$. Initially, the platform is located in the center of the structure, and the initial velocity is zero. Simulation time is $t=10$ seconds. The platform moves in 6-DOF motion following the desired trajectory.

\subsection{Analysis of the results}

Figure 6, 7 illustrate the translational and rotational motion of the platform. The platform motion is achieved by adjusting the length of cables. The length of cables is controlled by the LMI-based controller presented in Section 3.3. The average of the root mean square errors (RMSE) of position and rotation were $5.34 \times 10^{-4}$ and 


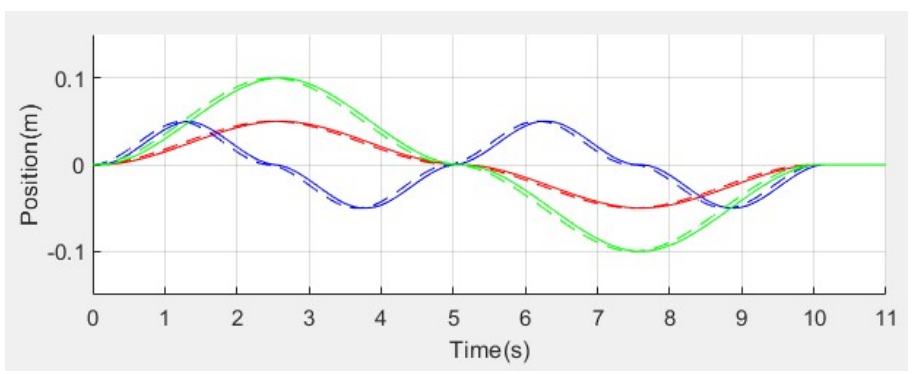

Figure 6: Translational motion of the platform. Motion in $x$-axis(surge) is red, in $y$-axis(sway) is blue, and in $z$-axis(heave) is green. Dashed lines are desired positions, and solid lines are controlled positions. Surge and sway changes from -5 to $5 \mathrm{~cm}$, heave changes -10 to $10 \mathrm{~cm}$. Surge and heave periods are 10 seconds, and sway is 5 seconds.

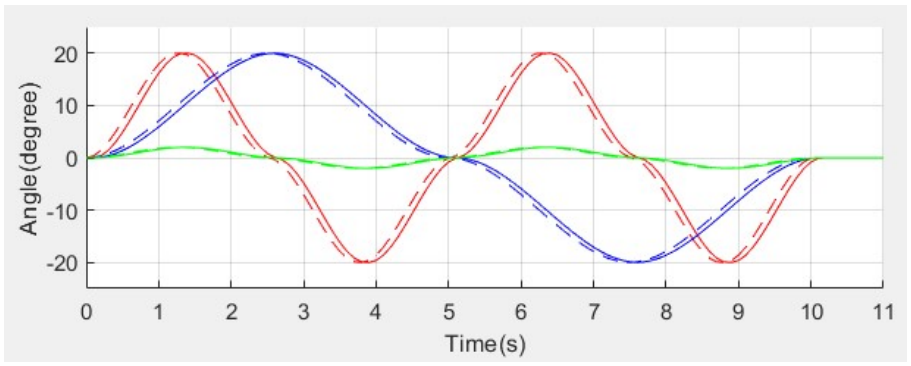

Figure 7: Rotational motion of the platform. Rotation in $x$-axis (roll) is red, in $y$-axis (pitch) is blue, and in $z$-axis (yaw) is red. Dashed lines are desired rotations, and solid lines are controlled rotations. Roll and pitch changes from $\mathbf{- 2 0}$ to 20 degrees, yaw changes -2 to 2 degrees. Roll and yaw periods are 5 seconds, and pitch is 10 seconds.

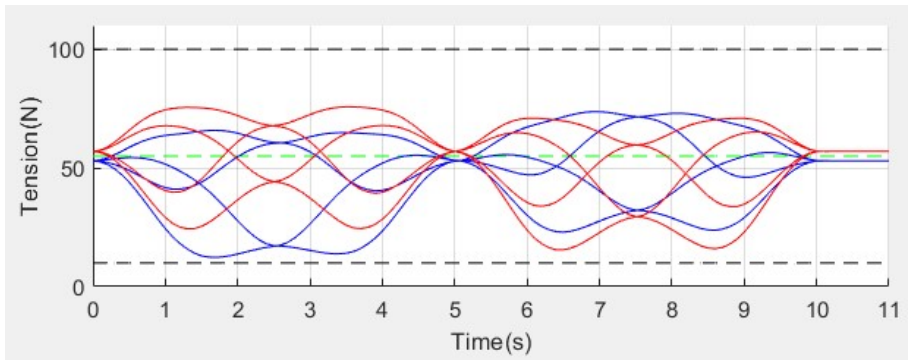

Figure 8: Optimized cable tension. The blue lines are lower cables and the red lines are upper cable. Black dashed lines are the minimum tension limit $(10 \mathrm{~N})$, and the maximum tension limit $(100 \mathrm{~N})$. The green line is the mean value of the minimum and the maximum tension limits $(55 \mathrm{~N})$.

$2.85 \times 10^{-3}$, showing that the LMI-based controller robustly controls the system.

The standard deviation optimization method discussed in Section 2.3 optimized cable tensions at each platform position. The minimum cable tension was $12.32 \mathrm{~N}$, and the maximum tension was $75.76 \mathrm{~N}$. Figure 8 shows that cable tensions are bounded in the minimum and the maximum tensions while the platform is moving. As shown in Figure 9, corresponding cable lengths were calculated by equation 7 ).

Defining the possible maximum tension margin as the difference between the maximum and the minimum tension limits, the percentage of tension margins to the possible maximum tension margin is shown in Figure 10. The lower bound tension margin percentage was from $2.58 \%$ to $47.79 \%$ and the upper bound tension margin was from $26.93 \%$ to $47.79 \%$.

These results verify that the LMI-based controller design controls the platform in 6-DOF motion robustly, and the optimization method maintains positive tension margins while controlling the platform.

\section{CONCLUSION}

In this paper, the tension optimization of an LMI-based controlled 6DOF cable-driven boat motion simulator was presented. This study focuses on verifying the feasibility of an LMI-based controller on the linearized system model and the tension optimization of the model. 


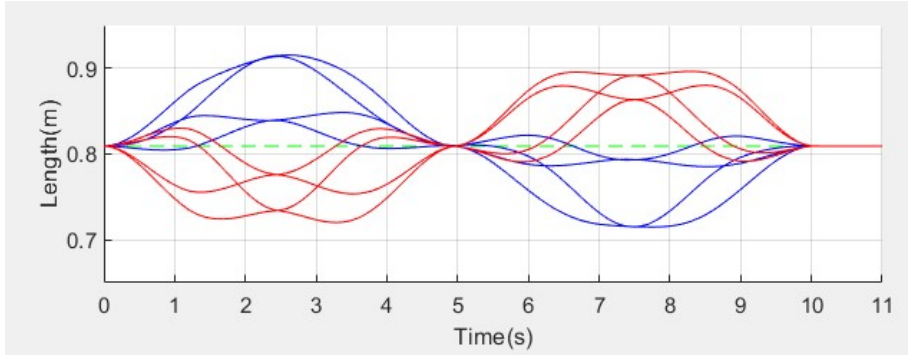

Figure 9: Cable length corresponding to cable tensions in Figure 8. The blue lines are lower cables, and the red lines are upper cables. The green line is a cable length at the origin..

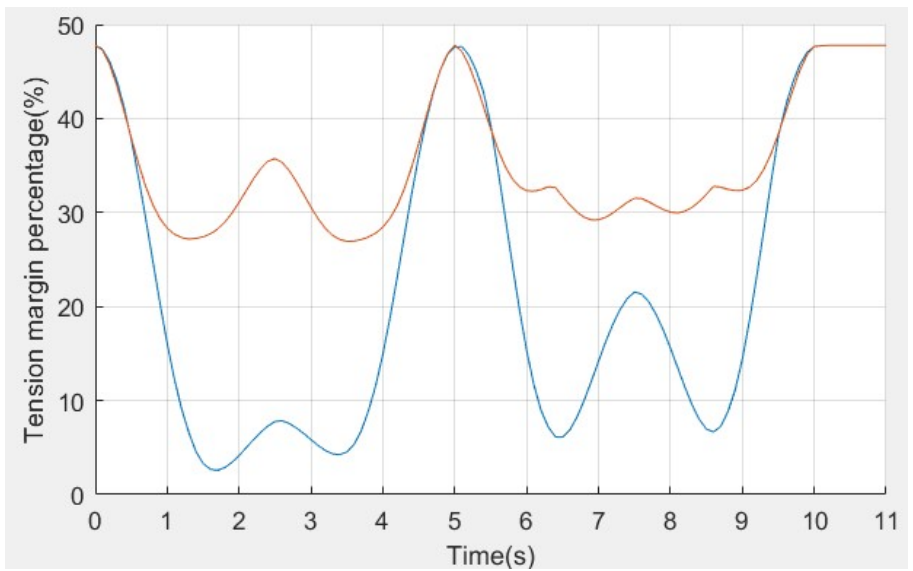

Figure 10: Tension margin percentage to the possible maximum tension margin $(90 \mathrm{~N})$. The red line is the upper bound tension margin, and the blue line is the lower bound tension margin.

Eight cables were connected to a platform that moves in 6-DOF motion and the platform replicates the motion of the boat on the sea. Leveraging the tensegrity system theory, statics and dynamics of the 6-DOF cable-driven system were analyzed, and an LMI-based feedback control model was developed. An LMI-based feedback controller is designed based on the time-variant linearized system that changes by the equilibrium position and cable lengths. The 6-DOF cable-driven system simulation shows that the LMI-based controller robustly controls the motion of the platform following the desired trajectory. The desired trajectory includes 6-DOF motions, but the simulator cannot rotate in z-axis (yaw) compared to the rotation in $\mathrm{x}$ (roll) or $\mathrm{y}$-axis (pitch) due to the cable attachment points on the platform. This problem can be solved by crossed cable attachment points on the platform. Adjusting the attachment points and redesigning the platform for larger workspace will be discussed in future work. Additionally, the standard deviation optimization method maintained cable tensions within the tension limit boundaries. The corresponding cable length was computed from the optimized tension for the actual motor control. Tension margins were positive, indicating that all cables are in positive tensions while the platform is moving. We plan to build an experimental 6-DOF cable-driven boat motion simulator with tension sensors to validate the simulation results. The actual data of ship motions will be used to generate desired trajectories.

\section{REFERENCES}

[1] K. Talke, D. Drotman, N. Stroumtsos, M. de Oliveira and T. Bewley. 2019. Design and Parameter Optimization of a 3-PSR Parallel Mechanism for Replicating Wave and Boat Motion. 2019 International Conference on Robotics and Automation (ICRA). pp. 7955-7961. doi: 10.1109/ICRA.2019.8793473.

[2] Bhaskar Dasgupta and T.S. Mruthyunjaya. 2000. The Stewart platform manipulator: a review. Mechanism and Machine Theory. Volume 35. Issue 1. Pages 15-40. ISSN 0094-114X, https://doi.org/10.1016/S0094-114X(99)00006-3.

[3] P. Miermeister et al. 2016. The CableRobot simulator large scale motion platform based on cable robot technology. 2016 IEEE/RSJ International Conference on Intelligent Robots and Systems (IROS). pp. 3024-3029. doi: 10.1109/IROS.2016.7759468.

[4] J. Jung, J. Piao, S. Park, J. Park and S. Y. Ko. 2016. Analysis of cable tension of high speed parallel cable robot: High speed position tracking of winch. 2016 16th International Conference on Control, Automation and Systems (ICCAS). pp. 1053-1056. doi: 10.1109/ICCAS.2016.7832439.

[5] A. Ghasemi, M. Eghtesad and M. Farid. 2008. Workspace analysis of redundant cable robots. 2008 World Automation Congress. pp. 1-6.

[6] X. Diao and O. Ma. 2006. Workspace Analysis of a 6-DOF Cable Robot for Hardware-in-the-Loop Dynamic Simulation. 2006 IEEE/RSJ International Conference on Intelligent Robots and Systems. pp. 4103-4108. doi: 10.1109/IROS.2006.281876.

[7] Nguyen, D.Q., and Gouttefarde, M. 2015. On the Improvement of Cable Collision Detection Algorithms. Mechanisms and Machine Science. pp. 29-40.

[8] Gallina, Paolo, Rossi, Aldo, and Williams, Robert. 2001. Planar Cable-DirectDriven Robots, Part II: Dynamics and Control. Proceedings of the ASME Design Engineering Technical Conference. 2. 10.1071/ASEG2001ab148.

[9] Zarebidoki, Mahmoud, Lotfavar, A., and Fahham, Hamid. 2011. Dynamic Modeling and Adaptive Control of a Cable-suspended Robot. Proceedings of the World Congress on Engineering 2011, WCE 2011. 3. 2469-2473.

[10] A. Aflakiyan, H. Bayani and M. T. Masouleh. 2015. Computed torque control of a cable suspended parallel robot. 2015 3rd RSI International Conference on Robotics and Mechatronics (ICROM). pp. 749-754. Tehran. 
[11] T. Bewley. 2017. Numerical renaissance. Renaissance Press. pp. 580-582.

[12] R. Mersi, S. Vali, M. S. haghighi, G. Abbasnejad and M. T. Masouleh. 2018. Design and Control of a Suspended Cable-Driven Parallel Robot with Four Cables. 2018 6th RSI International Conference on Robotics and Mechatronics (IcRoM). pp. 470-475. doi: 10.1109/ICRoM.2018.8657534.

[13] A. Z. Loloei, M. M. Aref and H. D. Taghirad. 2009. Wrench feasible workspace anal ysis of cable-driven parallel manipulators using LMI approach. 2009 IEEE/ASME International Conference on Advanced Intelligent Mechatronics. pp. 1034-1039. doi: 10.1109/AIM.2009.5229723.

[14] R. Skelton and M. de Oliveira. 2019. Tensegrity systems. Springer, pp. 17-19.

[15] T. Bewley. 2019. Stabilization of low-altitude balloon systems, Part 2: riggings with multiple taut ground tethers, analyzed as tensegrity systems. University of California, San Diego. Unpublished.

[16] B. Ahmed. 2009. LMI-based state-feedback control design for a wind generating power plant. Journal of Electrical Engineering: Volume 9 - Edition 4.

[17] B. Adamu, T. Nura, M. Mustapha, abd A. U. Sambo. 2020. Optimized state feedback control of quarter car active suspension system based on LMI algorithm. Journal of Physics: Conference Series.

[18] H. D. Abdulla and V. Snasel. 2008. Using Singular Value Decomposition (SVD) as a solution for search result clustering. 2008 International Conference on Innovations in Information Technology. pp. 302-306. doi: 10.1109/INNOVATIONS.2008.4781642.
[19] S. Boyd, and L. Vandenberghe. 2004. Convex optimization. Cambridge: Cambridge University Press

[20] M. Grant, and S. Boyd. 2009. CVX: Matlab software for disciplined convex programming, version 2.0 beta. http://cvxr.com/cvx.

[21] J. Begey, L. Cuvillon, M. Lesellier, M. Gouttefarde and J. Gangloff. 2019. Dynamic control of parallel robots driven by flexible cables and actuated by positioncontrolled winches. in IEEE Transactions on Robotics. vol. 35. no. 1. pp. 286-293.

[22] J. P. Hespanha. 2018. Linear systems theory: second edition. NED - New edition, 2 ed. Princeton University Press. pp. 12-30.

[23] H. Gritli, S. Belghith and A. Zemouche. 2019. LMI-based Design of Robust Static Output Feedback Controller for Uncertain Linear Continuous Systems. 2019 International Conference on Advanced Systems and Emergent Technologies (IC_ASET). pp. 243-248. doi: 10.1109/ASET.2019.8871044.

[24] M. de Oliveira. 2019. Linear control design [Class handout]. University of California, San Diego.

[25] V. Veselý. 2003. Robust output feedback controller design: LMI approach, IFAC proceedings volumes. Volume 36, Issue 8, ISSN 1474-6670. pp. 75-80. 2003.

[26] J. Lofberg. 2004. YALMIP: a toolbox for modeling and optimization in MATLAB. 2004 IEEE International Conference on Robotics and Automation (IEEE Cat. No.04CH37508). pp. 284-289. Taipei, Taiwan. 